\title{
Efeito de uma formulação hidrofílica de paromomicina tópica na leishmaniose cutânea em pacientes com contra-indicações de tratamento com antimonial pentavalente
}

\author{
Effect of a hydrophilic formulation of topical paromomycin on \\ cutaneous leishmaniasis among patients with contraindications for \\ treatment with pentavalent antimonials
}

\author{
Aline Marques dos Santos ${ }^{1}$, Elza Ferreira Noronha ${ }^{1}$, Lucas Antônio Miranda Ferreira ${ }^{2}$, \\ César Omar Carranza-Tamayo ${ }^{1}$, Elisa Cupolillo ${ }^{3}$ e Gustavo Adolfo Sierra Romero ${ }^{1}$
}

\begin{abstract}
RESUMO
Descrevem-se o efeito terapêutico e os eventos adversos associados com o uso tópico de paromomicina 10\% em gel na leishmaniose cutânea. Quinze pacientes com leishmaniose cutânea cumpriram os critérios de inclusão descritos a seguir: contra-indicação para o uso de antimoniato de meglumina, intradermorreação de Montenegro positiva e até quatro lesões ulceradas. A fórmula foi prescrita duas vezes ao dia por 20 dias. Quatorze pacientes estiveram disponíveis para a avaliação do desfecho terapêutico e a proporção de cura foi de 21,4\% (3/14), 50\% melhoraram até a epitelização completa e a proporção de falha foi de $28,6 \%$. Nove pacientes que não apresentaram cura inicialmente foram re-tratados. Oito receberam uma nova série de paromomicina tópica e um foi tratado com antimoniato de meglumina. Dois pacientes não receberam novo tratamento e tiveram melhora lenta e contínua. Cinco de oito pacientes retratados com paromomicina tópica alcançaram a cura clínica, e três apresentaram falha, incluindo um paciente que tinha apresentado melhora com o primeiro tratamento. Os eventos adversos foram leves e locais em 53,3\% dos pacientes e nunca levaram à suspensão do tratamento.
\end{abstract}

Palavras-chaves: Leishmaniose cutânea. Leishmania braziliensis. Paromomicina. Tratamento tópico.

\section{ABSTRACT}

The therapeutic effect of and adverse events associated with topical use of $10 \%$ paromomycin gel on cutaneous leishmaniasis are described. Fifteen patients with cutaneous leishmaniasis fulfilled the following inclusion criteria: contraindication for the use of meglumine antimoniate, positive Montenegro skin test and up to four ulcerated lesions. The formula was prescribed twice a day for 20 days. Fourteen patients were available for the therapeutic outcome evaluation. The cure rate was $21.4 \%$ (3/14); $50 \%$ improved as far as complete epithelialization; and the failure rate was $28.6 \%$. Nine patients who did not initially present cure were retreated. Eight received a new series of topical paromomycin and one was treated with meglumine antimoniate. Two patients did not receive any new treatment and had continuous slow improvement. Five out of the eight patients retreated with topical paromomycin achieved clinical cure, and three presented failure, including one patient who had shown any improvement with the first treatment. For $53.3 \%$ of the patients, the adverse events were mild and local and never led to treatment suspension.

Key-words: Cutaneous leishmaniasis. Leishmania braziliensis. Paromomycin. Topical treatment.

0 tratamento ambulatorial da leishmaniose tegumentar no Brasil, realizado com o antimoniato de meglumina (AM) durante 20 a 30 dias, apresenta toxicidade relativamente frequiente. Os efeitos mais relevantes são mialgias, artralgias, anorexia, febre, alterações eletrocardiográficas e alterações bioquímicas da função hepática, renal e pancreática e impedem a aplicação segura em pessoas com algumas doenças crônicas e em grávidas ${ }^{71721}$. A paromomicina (PA), um antibiótico aminoglicosídeo extraído de culturas do Streptomyces rimosus var.paromomicinus, éidêntica à aminosidina produzida pelo Streptomyces chrestomyceticus. Este fármaco tem ação leishmanicida comprovada e atualmente se encontra em fase de desenvolvimento avançado para o tratamento da leishmaniose visceral ${ }^{24}$.

\footnotetext{
1. Núcleo de Medicina Tropical, Faculdade de Medicina, Universidade de Brasília, Brasília, DF. 2. Departamento de Produtos Farmacêuticos, Universidade Federal de Minas Gerais, Belo Horizonte, MG. 3. Laboratório de Imunologia do Instituto Oswaldo Cruz, Fundação Instituto Oswaldo Cruz, Rio de Janeiro, RJ.

Apoio financeiro: Fundação de Apoio à Pesquisa do Distrito Federal - FAPDF (Processo PRONEX/FAPDF 193.000-114/2004); Fundação de Empreendimentos Científicos e Tecnológicos - FINATEC; Programa de Bolsas de Iniciação Científica do Conselho Nacional de Desenvolvimento Científico e Tecnológico - CNPq/MCT; Programa de bolsas de Pós-graduação da Coordenação de Aperfeiçoamento de Pessoal de Nível Superior - CAPES/MEC.

Endereço para correspondência: Dr. Gustavo Romero. Núcleo de Medicina Tropical/UnB. Caixa postal 04517, Campus Universitário, $70904-970$ Brasília, DF.

Tel: 5561 3273-5008; Fax: $55613273-2811$.

e-mail: gromero@unb.br

Recebido para publicação em 11/02/2008

Aceito em 01/09/2008
} 
Compostos com PA para uso tópico foram testados em vários países com resultados variáveis em ensaios clínicos de fase II e III $^{2} 3141520$. Essa variabilidade da resposta ao tratamento tópico com PA poderia estar condicionada ao uso de diferentes veículos farmacêuticos e à espécie de Leishmania ${ }^{11}$.

A permeação cutânea in-vitro da PA, avaliada em pele excisada de camundongos glabros na ausência do estrato córneo, foi muito maior, cerca de 30 vezes, a partir de formulações hidrofílicas (creme ou gel) em comparação com uma pomada de vaselina ${ }^{12}$. Também, a atividade leishmanicida, avaliada em camundongos BALB/c experimentalmente infectados com Leishmania major, foi maior com as formulações hidrofílicas em comparação com a pomada, sendo que ambas as preparações foram capazes de promover a cicatrização das lesões ${ }^{4} 8$. Estudos em animais infectados com Leishmania (Leishmania) amazonensis $e$ Leishmania (Viannia) braziliensis demonstraram que a formulação hidrofílica de PA tópica teve resultados satisfatórios quando comparada ao AM, administrado por via sistêmica ${ }^{12}$.

Considerando que um estudo de fase I em humanos voluntários sadios seria uma proposta inviável, uma vez que a aplicação tópica da PA pressupõe a falta da integridade da barreira cutânea para atuar, foi desenvolvido um estudo aberto não controlado para avaliar os efeitos terapêuticos e colaterais de uma formulação hidrofílica em pacientes que apresentaram contra-indicações para o uso de AM. 0 objetivo deste trabalho foi avaliar o efeito da aplicação tópica de uma formulação hidrofílica de PA como alternativa menos tóxica em um grupo de pacientes com maior vulnerabilidade à toxicidade pelo AM. Uma vez que a quantidade de PA absorvida através da pele é menor do que a aplicada por via parenteral nas doses convencionais utilizadas anteriormente para o tratamento sistêmico da leishmaniose cutânea ${ }^{518}$, a aplicação tópica surge como uma alternativa terapêutica com menor toxicidade.

\section{PACIENTES E MÉTODOS}

O estudo foi realizado no Centro de Saúde da comunidade de Corte de Pedra, município de Presidente Tancredo Neves, Bahia, que funciona como unidade de referência para o tratamento de leishmaniose na região. Estudos recentes continuam demonstrando que o parasita predominante é Leishmania (Viannia) braziliensis $^{17}$. 0 período de recrutamento dos pacientes foi abril de 2004 a março de 2005, sendo incluídos 15 pacientes adultos, sem restrição de idade. Todos apresentavam até quatro lesões ulceradas na pele, de duas ou mais semanas de evolução; intradermorreação de Montenegro positiva; uma ou mais contraindicações para o uso de AM, tais como, gravidez, amamentação, idade avançada (>65 anos), hipersensibilidade ao uso de AM, hipertensão arterial, diabetes melitus, cardiopatias e nefropatias. Devido à toxicidade potencial da droga em estudo foram excluídos pacientes com história de hipersensibilidade a qualquer um dos componentes da formulação tópica; asmáticos ou com história de alergia a sulfitos; portadores de nefropatias agudas ou crônicas; doenças do aparelho auditivo com manifestações clínicas; uso de drogas nefrotóxicas; insuficiência venosa crônica ou insuficiência arterial sintomática; uso de drogas leishmanicidas nos três meses anteriores à consulta, exceto quando foram constatados efeitos adversos agudos durante a primeira e única dose de tratamento com AM; ou gestação no $1^{\circ}$ trimestre.

Foram realizadas a intradermorreação de Montenegro e a cultura do aspirado de lesões cutâneas e de linfonodos infartados, segundo os métodos previamente descritos ${ }^{19}$. Os parasitas isolados foram identificados utilizando-se a eletroforese de enzimas conforme metodologia previamente descrita ${ }^{6}$. Também foram realizadas biópsias da borda da lesão para extrair DNA para realizar a reação em cadeia pela polimerase (PCR), tendo como alvo de amplificação a região conservada de 120 pares de bases do k-DNA do parasito. Para a extração do DNA foi utilizado o Genomic Prep ${ }^{T M}$ Tissue and Cells DNA Isolation Kit (Amershan Biosciences, UK) de acordo com as instruções do fabricante e a reação de amplificação foi realizada seguindo o protocolo publicado anteriormente ${ }^{16}$.

A formulação hidrofílica, compostapor gel dehidroxietilcelulose contendo PA a $10 \%$ e cloreto de metilbenzetônio a $0,1 \%$, foi prescrita para aplicação nas lesões duas vezes ao dia por vinte dias. Realizou-se acompanhamento clínico, com aferição dos diâmetros sagital e transversal das lesões ulceradas a cada mês por período de 12 semanas após o término do tratamento. Aárea ulcerada foi estimada em $\mathrm{cm}^{2}$ utilizando-se a fórmula: área = (diâmetro transversal/2) X (diâmetro sagital/2) X 3,14. 0 efeito terapêutico foi classificado por critérios clínicos de cura, melhora e falha avaliados após um período de 12 semanas depois de finalizado o tratamento. A cura foi caracterizada como cicatrização completa e ausência de eritema, edema e descamação; a melhora foi definida como epitelização completa das úlceras com persistência dos demais sinais flogísticos; e a falha caracterizou-se pela ausência de epitelização completa das lesões, piora dos sinais inflamatórios, aumento do tamanho da úlcera ou aparecimento de lesões satélites em relação às lesões primárias.

Para os pacientes que não curaram, foi prescrita segunda série de tratamento com a PA tópica, sendo observado o mesmo protocolo descrito acima, ou AM quando a contra-indicação para o seu uso foi temporária.

Considerações éticas. Todos os pacientes assinaram 0 termo de consentimento livre e esclarecido; os pacientes foram selecionados por meio da aplicação de critérios de inclusão dirigidos a reduzir a possibilidade de toxicidade local e sistêmica. O projeto cumpriu com a resolução 196/96 do Conselho Nacional de Saúde sobre Pesquisa Envolvendo Seres Humanos e foi aprovado pelo Comitê de Ética em Pesquisa da Faculdade de Medicina da Universidade de Brasília (processo número: 041/02).

\section{RESULTADOS}

Foram incluídos 15 pacientes, todos apresentaram intradermorreação de Montenegro positiva, seis do sexo masculino e nove do sexo feminino, com mediana de idade de 56 anos (intervalo de 19 a 88 anos). As contra-indicações para o uso de AM foram: 46,7\% (7/15) hipertensão arterial descompensada e sem 
tratamento; $13,3 \%$ (2/15) gravidez; 13,3\% (2/15) amamentação; $13,3 \%(2 / 15)$ efeitos adversos graves ao AM; 6,7\% (1/15) idade avançada; e 6,7\% (1/15) angina pectoris. A mediana do número de lesões foi de 1 (intervalo de 1 a 4 ), sendo que oito pacientes apresentaram apenas uma lesão, quatro apresentaram duas lesões, dois apresentaram três lesões e um apresentou quatro lesões. A mediana do tempo de evolução da doença na primeira consulta, foi de sete semanas (intervalo de 2 a 20 semanas) e a mediana da área total ulcerada foi de $3,9 \mathrm{~cm}^{2}$ (intervalo de 0,8 a $17,7 \mathrm{~cm}^{2}$ ). Quanto à localização das úlceras, oito pacientes apresentaram lesões nos membros inferiores; três nos membros superiores; um no tronco; um em membro inferior e tronco; um em membro superior e tronco; e um na cabeça, pescoço, membro superior e tronco. Dentre os 15 pacientes avaliados, apenas oito apresentaram acometimento linfático, seis dos quais tiveram adenomegalia, um linfangite e um paciente ambos os achados.

O diagnóstico parasitológico foi confirmado, por meio de cultura de pele ou linfonodos em cinco $(33,3 \%)$ pacientes. Os parasitos isolados de cinco pacientes foram identificados por meio de eletroforese de enzimas como Leishmania (Viannia) braziliensis. Quatorze pacientes apresentaram o exame de PCR positivo. Somente uma paciente teve a reação de Montenegro como único exame de diagnóstico positivo.

A mediana do tempo de tratamento foi de 30 dias (intervalo de 20 a 57 dias), sendo que apenas três pacientes seguiram a indicação de aplicar o tratamento rigorosamente por 20 dias. As irregularidades observadas foram as seguintes: em dez casos a medicação foi usada por mais tempo que o preconizado no protocolo; e, em um caso, a medicação foi interrompida por seis dias durante o parto e puerpério imediato. Uma paciente foi excluída da avaliação de efeito terapêutico porque foi submetida ao tratamento com AM em outra unidade de saúde imediatamente depois de finalizado o tratamento com PA, o que constituiu cointervenção. A taxa de cura foi de 21,4\% (3/14), a de melhora foi de 50\% (7/14) e houve 28,6\% (4/14) de falha. Dos 11 pacientes que não curaram inicialmente, oito receberam uma nova série de PA tópica, um recebeu AM e dois foram observados sem novos tratamentos. Cinco, dos oito que receberam PA tópica, curaram e três cumpriram critérios de falha terapêutica, incluindo um paciente que havia melhorado após a primeira série de tratamento. Dos três pacientes que apresentaram falha terapêutica, um abandonou o acompanhamento após a segunda série de tratamento com PA tópica; um recebeu azitromicina oral por 20 dias e abandonou 0 acompanhamento; e um recebeu AM evoluindo para a cura três meses depois. A mediana do tempo total de acompanhamento clínico, desde a primeira consulta até a última avaliação foi de 201 dias (intervalo de 122 a 279 dias). Os dados completos dos pacientes estão detalhados individualmente na Tabela 1 .

Os eventos adversos associados à PA tópica foram avaliados nos 15 pacientes tratados constatando-se que 46,7\% (7/15) não apresentaram queixas; $13,3 \%$ (2/15) relataram prurido; $20 \%$ (3/15) tiveram sensação de ardor local durante a aplicação do medicamento; e 20\% (3/15) apresentaram eritema perilesional, dor, edema e sensação de ardor local.

Tabela 1 - Características clínicas e laboratoriais de 15 pacientes com leishmaniose cutânea localizada tratados com uma formulação tópica de paromomicina 10\% em Corte de Pedra, Município de Presidente Tancredo Neves, Babia. 2004-2005.

\begin{tabular}{|c|c|c|c|c|c|c|c|c|c|c|c|c|}
\hline \multirow[b]{2}{*}{ Paciente } & \multirow{2}{*}{$\begin{array}{l}\text { Idade } \\
\text { (anos) }\end{array}$} & \multirow[b]{2}{*}{ Sexo } & \multirow[b]{2}{*}{ IMC } & \multirow{2}{*}{$\begin{array}{l}\text { Lesões } \\
\left(\mathrm{n}^{\mathrm{o}}\right)\end{array}$} & \multirow{2}{*}{$\begin{array}{c}\text { Área total } \\
\text { ulcerada }\left(\mathrm{cm}^{2}\right)\end{array}$} & \multirow{2}{*}{$\begin{array}{c}\text { Duração da } \\
\text { doença (sem) }\end{array}$} & \multicolumn{2}{|c|}{$\begin{array}{c}\text { Diagnóstico da } \\
\text { leishmaniose }\end{array}$} & \multirow{2}{*}{$\begin{array}{c}\text { Tempo de tratamento } \\
\text { inicial (dias) }\end{array}$} & \multirow{2}{*}{$\begin{array}{l}\text { Resultado do } \\
\text { tratamento } \\
\text { inicial }\end{array}$} & \multirow[b]{2}{*}{ Retratamento } & \multirow{2}{*}{$\begin{array}{l}\text { Resultado } \\
\text { final }\end{array}$} \\
\hline & & & & & & & cultura & PCR & & & & \\
\hline 1 & 19 & $\mathrm{~F}$ & 22,3 & 2 & 11,7 & 20 & + & + & 35 & melhora & $\mathrm{PA}$ & cura \\
\hline 2 & 78 & M & 22,6 & 2 & 0,8 & 8 & + & + & 33 & melhora & $\mathrm{PA}$ & cura \\
\hline 3 & 52 & $\mathrm{~F}$ & 23,8 & 3 & 2,7 & 9 & - & + & 26 & melhora & $\mathrm{PA}$ & cura \\
\hline 4 & 65 & M & 24,4 & 1 & 1,4 & 8 & - & + & 30 & cura & nenhum & cura \\
\hline 5 & 74 & $\mathrm{~F}$ & 23,5 & 1 & 1,8 & 12 & - & - & 20 & melhora & $\mathrm{PA}$ & falha \\
\hline 6 & 27 & M & 22,0 & 1 & 3,9 & 6 & - & + & 20 & falha & $\mathrm{PA}$ & falha \\
\hline 7 & 88 & M & 20,3 & 1 & 17,7 & 8 & - & + & 28 & cura & nenhum & cura \\
\hline 8 & 19 & $\mathrm{~F}$ & 23,1 & 1 & 7,1 & 2 & + & + & 26 & melhora & $\mathrm{PA}$ & cura \\
\hline 9 & 19 & $\mathrm{~F}$ & 21,0 & 2 & 7,9 & 6 & + & + & 20 & falha & PA & falha \\
\hline 10 & 57 & F & 23,3 & 1 & 0,8 & 4 & - & + & 36 & melhora & $\mathrm{PA}$ & cura \\
\hline 11 & 47 & $\mathrm{~F}$ & 22,7 & 3 & 2,0 & 4 & - & + & 52 & cura & nenhum & cura \\
\hline 12 & 56 & $\mathrm{~F}$ & 17,6 & 2 & 4,1 & 9 & - & + & 30 & abandono & $\mathrm{AM}$ & cura \\
\hline 13 & 40 & $\mathrm{~F}$ & 22,0 & 1 & 1,3 & 8 & - & + & 57 & melhora & nenhum & melhora \\
\hline 14 & 61 & M & 23,0 & 1 & 5,7 & 6 & - & + & 30 & falha & $\mathrm{AM}$ & melhora \\
\hline 15 & 80 & M & 21,7 & 4 & 5,0 & 6 & + & + & 45 & falha & nenhum & melhora \\
\hline
\end{tabular}

IMC: índice de massa corporal, PCR: reação em cadeia da polimerase, F: feminino, M: Masculino, PA: paromomicina tópica, AM: antimoniato de meglumina.

\section{DISCUSSÃo}

A inclusão de 15 pacientes com contra-indicações para 0 uso de AM, no período de 12 meses de estudo, demonstra a importância da limitação do uso do antimonial por diversos motivos e reafirma a relevância da necessidade de um tratamento alternativo e menos tóxico para a leishmaniose cutânea.
No presente estudo, a resposta à formulação hidrofílica de PA tópica mostrou-se benéfica, já que se observou evolução para a cura ou melhora na maioria dos pacientes. Com o re-tratamento, evidenciou-se incremento desse benefício, já que o número de pacientes curados aumentou. No entanto, foram observados episódios de ausência total de resposta à PA tópica, o que raramente é observado quando os pacientes são submetidos ao tratamento com $\mathrm{AM}^{17}$. 
A adesão dos pacientes à prescrição, que deveria ser apenas de 20 dias, foi inadequada, o que revela a dificuldade do tratamento tópico auto-administrado por tempo definido quando se espera que haja persistência de alguns sinais, tais como a permanência de feridas abertas, após 20 dias de uso. A maioria dos pacientes prolongou por conta própria o tempo de uso da PA tópica, fenômeno facilitado pela ausência de efeitos adversos locais importantes.

0 tempo até 0 aparecimento do efeito terapêutico, expresso pela cura ou melhora das lesões parece ser mais longo que o observado com o tratamento com AM quando se avalia a experiência com controles históricos na mesma localidade ${ }^{17}$, confirmando os achados relatados por outros autores ${ }^{1}$. Na Tunísia também se observou melhora das lesões ao final do tratamento com PA tópica, embora não tenha havido cura significativa ${ }^{20}$.

Em relação aos efeitos adversos, observou-se que não foram relevantes nem freqüentes na amostra estudada, não estando associados, em nenhum momento, à interrupção do tratamento.

A formulação testada de PA mostrou melhor perfil de tolerância, devido a uma menor intensidade de efeitos adversos locais, do que aquela observada com outras formulações tais como as que contêm cloreto de metilbenzetônio que foram relatadas na literatura capazes de produzir vesículas ${ }^{22} 23$.

Apesar do estudo ser do tipo aberto e não controlado com número pequeno de pacientes, foi possível observar o efeito terapêutico da formulação hidrofílica de PA tópica com perfil de tolerância aceitável. Contudo, o resultado sugere que este regime parece requerer tempo de aplicação maior do que vinte dias para que haja a cura efetiva.

0 presente relato é o primeiro mostrando o efeito terapêutico desta formulação hidrofílica de PA em um subgrupo especial de pacientes procedentes de uma área de transmissão de Leishmania (Viannia) braziliensis, para os quais existem poucas alternativas terapêuticas para uso em regime ambulatorial. Um dos pacientes que não teve resposta com a paromomicina foi tratado com azitromicina que tem sido relatada com algum efeito sobre a leishmaniose $e^{25}$. Os resultados encorajam a realização de novos ensaios controlados em uma população de doentes menos selecionada que permita estimar a eficácia da droga. 0 presente estudo apresenta a limitação de um número pequeno de observações que impede explorar o papel da localização, do tempo de evolução da doença e de outros fatores relevantes sobre o desfecho terapêutico estudado. É importante considerar que futuros ensaios clínicos devem contemplar a possibilidade de ação sinérgica da formulação hidrofílica de PA associada a doses menores de AM ou a outras drogas administradas pela via oral, utilizando-se uma abordagem similar àquela aplicada ao desenvolvimento de outras formulações de paromomicina ${ }^{2223}$. 0 estudo da combinação da PA tópica com outras drogas, utilizando-se modelos animais ${ }^{1013}$, deve ser aprofundado em busca de um espectro terapêutico amplo para os pacientes com contra-indicação a AM. Considerando a diversidade de espécies de Leishmania associadas com a doença cutânea também serão necessárias pesquisas dirigidas a investigar a sensibilidade dessas espécies à PA, e a possível influência dos padrões de sensibilidade in vitro com o tipo de desfecho clínico observado com o tratamento, sobretudo, nos casos onde se observa falha precoce.

\section{AGRADECIMENTOS}

Lília Firoozmand pelo auxílio nos procedimentos de identificação dos isolados por meio de eletroforese de enzimas, Tércio Pereira Rodrigues pela assistência técnica no preparo do meio de cultura, Marlete Silva Santos pelo auxílio no cuidado aos pacientes e Renata Ribero de Sousa pelo auxílio na realização da PCR.

\section{REFERÊNCIAS}

1. Armijos RX, Weigel MM, Calvopiña M, Mancheno M, Rodriguez R. Comparison of the effectiveness of two topical paromomycin treatments versus meglumine antimoniate for New World cutaneous leishmaniasis. Acta Tropica 91:153-160, 2004.

2. Asilian A, Jalayer T, Whitwortth JAG, Ghasemi RL, Nilforooshzadeh M, Olliaro P. A randomized, placebo-controlled trial of two-week regimen of aminosidine (paramomycin) ointment for treatment of cutaneous leishmaniasis in Iran. The American Journal of Tropical Medicine and Hygiene 53: 648-651,1995.

3. Bryceson ADM, Murphy A, Moody AH. Treatment of Old-World cutaneous leishmaniasis with aminosidine ointment - results of an open study in London. Transactions of the Royal Society of Tropical Medicine and Hygiene 88: 226-228, 1994.

4. Carter KC, Alexander J. Studies on the topical treatment of experimental cutaneous leishmaniasis: the therapeutic effect of methylbenzetonium chloride and the aminoglycosides, gentamicin and paramomycin. Annals of Tropical Medicine and Parasitology 83: 233-239, 1989.

5. Correia D, Macêdo VO, Carvalho EM, Barral A, Magalhães AV, Abreu MV, Orge ML, Marsden P. Comparative study of meglumine antimoniate, pentamidine isethionate and aminosidine sulfate in the treatment of primary skin lesions caused by Leishmania (Viannia) braziliensis. Revista da Sociedade Brasileira de Medicina Tropical 29: 447-453, 1996.

6. Cupolillo E, Grimaldi Jr G, Momen H. A general classification of New World Leishmania using numerical zymotaxonomy. The American Journal of Tropical Medicine and Hygiene 50: 296-311, 1994.

7. Deps PD, Viana MC, Falqueto A, Dietze R. Comparative assessment of the efficacy and toxicity of N-methyl-glucamine and BP88 sodium stibogluconate in the treatment of localized cutaneous leishmaniasis. Revista da Sociedade Brasileira de Medicina Tropical 33: 535-543, 2000.

8. El-On J, Hamburguer AD. Topical treatment of New and Old World cutaneous leishmaniasis in experimental animals. Transactions of the Royal Society of Tropical Medicine and Hygiene 81: 734-737, 1987.

9. El-On J, Jacobs GP, Witztum E, Creenblat CL. Development of topical treatment for cutaneous leishmaniasis caused by Leishmania major in experimental animals. Antimicrobial Agents and Chemotherapy 26: 745-751, 1984.

10. Fernandes AP, Carvalho FA, Tavares CA, Santiago HC, Castro GA, Tafuri WL, Ferreira LA. Combined interleukin-12 and topical chemotherapy for established leishmaniasis drastically reduces tissue parasitism and relapses in susceptible mice. Journal of Infectious Diseases 183: 1646-1652, 2001.

11. Gomes SFO, Nunan EA, Ferreira LAM. Emulsões múltiplas A/O/A contendo paromomicina para o tratamento tópico da leishmaniose cutânea: obtenção, caracterização e estabilidade. Revista Brasileira de Ciências Farmacêuticas 36: $277-284,2000$.

12. Gonçalves GS, Fernandes AP, Souza RCC, Cardoso JE, Silva FO, Maciel FC, Rabello A, Ferreira LAM. Activity of a paromomycin hydrophilic formulation for topical treatment of infections by Leishmania (Leishmania) amazonensis and Leishmania (Viannia) braziliensis. Acta Tropica 93: 161-167, 2005. 
13. Grogl M, Schuster BG, Ellis WY, Berman JD. Successful topical treatment of murine cutaneous leishmaniasis with a combination of paromomycin (aminosidine) and gentamicin. Journal of Parasitology 85: 354-359, 1999

14. Krause G, Kroeger A. Topical treatment of American cutaneous leishmaniasis with paromomycin and methylbenzethonium chloride: a clinical study under field conditions in Ecuador. Transactions of the Royal Society of Tropical Medicine and Hygiene 88: 92-94, 1994.

15. Neva FA, Ponce C, Ponce E, Kreutzer R, Modabber F, Olliaro P. Non-ulcerative cutaneous leishmaniasis in Honduras fails to respond to topical paromomycin. Transactions of the Royal Society of Tropical Medicine and Hygiene 91: 473-475, 1997.

16. Romero GAS, Guerra MV, Paes MG, Cupolillo E, Toaldo CB, Macêdo VO, Fernandes 0 . Sensitivity of the polymerase chain reaction for the diagnosis of cutaneous leishmaniasis due to Leishmania (Viannia) guyanensis. Acta Tropica 79: 225229, 2001.

17. Romero GAS, Guerra MVF, Paes MG, Macedo VO. Comparison of cutaneous Leishmaniasis due to Leishmania (Viannia) braziliensis and L. (V.) guyanensis in Brazil: therapeutic response to meglumine antimoniate. The American Journal of Tropical Medicine and Hygiene 65: 456-465, 2001.

18. Romero GAS, Lessa HA, Macêdo VO, Carvalho EM, Barral A, Magalhães AV, Orge MG, Abreu MV, Marsden PD. Open therapeutic study with aminosidine sulfate in mucosal leishmaniasis caused by Leishmania (Viannia) braziliensis Revista da Sociedade Brasileira de Medicina Tropical 29: 557-565, 1996.
19. Romero GAS, Sampaio RNR, Macêdo VO, Marsden PD. Sensitivity of lymph node aspiration in localized cutaneous leishmaniasis due to Leishmania (Viannia) braziliensis. Memórias do instituto Oswaldo Cruz 94: 509-511, 1999.

20. Salah AB, Zakraqui H, Zaatour A, Ftaiti A, Zaafouri B, Garraoui A, Olliaro PL, Dellagi K, Ismail RB. A randomized, placebo-controlled trial in Tunisia treating cutaneous leishmaniasis with paromomycin ointment. The American Journal of Tropical Medicine and Hygiene 53: 162-166, 1995.

21. SaldanhaAC, Romero GA, Guerra C, Merchan-Hamann E, Macedo VO. Comparative study between sodium stibogluconate BP 88 and meglumine antimoniate in cutaneous leishmaniasis treatment. II. Biochemical and cardiac toxicity. Revista da Sociedade Brasileira de Medicina Tropical 33: 383-388, 2000.

22. Soto J, Fuya P, Herrera R, Berman J. Topical paromomycin/methylbenzethonium chloride plus parenteral meglumine antimoniate as treatment for American cutaneous leishmaniasis: controlled study. Clinical Infectious Diseases 26: 56-58, 1998.

23. Soto J, Hernandez N, Mejia H, Grogl M, Berman J. Successful treatment of New World cutaneous leishmaniasis with a combination of topical paromomycin/ methylbenzethonium chloride and injectable meglumine antimonite. Clinical Infectious Diseases 20: 47-51, 1995.

24. Sundar S, Jha TK, Thakur CP, Sinha PK, Bhattacharya SK. Injectable paromomycin for visceral leishmaniasis in India. New England Journal of Medicine 356: 2571 $2581,2007$.

25. Teixeira AC, Paes MG, Guerra J0, Prata A, Silva-Vergara ML. Low efficacy of azithromycin to treat cutaneous leishmaniasis in Manaus, AM, Brazil. Revista do Instituto de Medicina Tropical de São Paulo 49: 235-238, 2007. 